\title{
On Singular Interval-Valued Iteration Groups
}

\author{
Marek C. Zdun * \\ Institute of Mathematics, Pedagogical University of Cracow, Kraków, Poland
}

Let $I=(a, b)$ and $L$ be a nowhere dense perfect set containing the ends of the interval I and let $\varphi: I \rightarrow \mathbb{R}$ be a non-increasing continuous surjection constant on the components of $I \backslash L$ and the closures of these components be the maximal intervals of constancy of $\varphi$. The family $\left\{F^{t}, t \in \mathbb{R}\right\}$ of the interval-valued functions $F^{t}(x):=\varphi^{-1}[t+\varphi(x)], x \in I$ forms a set-valued iteration group. We determine a maximal dense subgroup $T \subsetneq \mathbb{R}$ such that the set-valued subgroup $\left\{F^{t}, t \in T\right\}$ has some regular properties. In particular, the mappings $T$ э $t \rightarrow F^{t}(x)$ for $t \in T$ possess selections $f^{t}(x) \in F^{t}(x)$, which are disjoint group of continuous functions.

Keywords: iteration group, set-valued functions, simultaneous functional equations, Cantor set, singular Lebesgue function

\section{OPEN ACCESS}

Edited by:

Witold Jarczyk,

University of Zielona Góra, Poland

Reviewed by:

Ludwig Reich

University of Graz, Austria

Krzysztof Cieplinski,

AGH University of Science and

Technology, Poland

*Correspondence:

Marek C. Zdun

mczdun@up.krakow.pl

Specialty section:

This article was submitted to

Dynamical Systems,

a section of the journal

Frontiers in Applied Mathematics and

Statistics

Received: 24 May 2016

Accepted: 30 August 2016

Published: 13 September 2016

Citation:

Zdun MC (2016) On Singular Interval-Valued Iteration Groups.

Front. Appl. Math. Stat. 2:13. doi: 10.3389/fams.2016.00013

\section{INTRODUCTION}

A family of functions $\left\{f^{t}: I \rightarrow I, t \in \mathbb{R}\right\}$ such that $f^{t} \circ f^{s}=f^{t+s}, t, s \in \mathbb{R}$ is said to be an iteration group, however a family of set-valued functions $\left\{F^{t}: I \rightarrow 2^{I}, t \in \mathbb{R}\right\}$ such that $F^{t} \circ F^{s}=F^{t+s}, t, s \in \mathbb{R}$ is said to be a set-valued iteration group (abbreviated to $s$ - $v$ iteration group). The notion of an iteration semigroup of set-valued functions was introduced and studied by Smajdor [1] and then studied in some classes of set-valued functions (see e.g., [2], [3], [4], [5]). The fundamental problem in the theory of multivalued iteration semigroups is the problem of existence and regularity properties of continuous selections. In this note we considered particular set-valued iteration groups whose values are the intervals or singletons. The presented results complete and generalize some of the topics from Zdun [6]. The considered s-v iteration groups have the very irregular properties. For every such s-v iteration group $\left\{F^{t}: I \rightarrow 2^{I}, t \in \mathbb{R}\right\}$ we find a special maximal additive subgroup $T \subset \mathbb{R}$ such that group $\left\{F^{t}: I \rightarrow 2^{I}, t \in T\right\}$ has several "regular" properties.

\section{MATERIALS AND METHODS}

Let $I=(a, b)$ and $\varphi: I \rightarrow \mathbb{R}$ be a surjection. Define the set-valued functions

$$
F^{t}(x):=\varphi^{-1}[\varphi(x)+t], \quad t \in \mathbb{R}, \quad x \in I .
$$

The surjection $\varphi$ is said to be the generating function of the family $\left\{F^{t}\right\}$.

THEOREM 1

The family $\left\{F^{t}: I \rightarrow 2^{I}\right\}$ is a set-valued iteration group, i.e.,

$$
F^{t} \circ F^{s}=F^{t+s}, \quad t, s \in \mathbb{R},
$$


where

$$
F^{t} \circ F^{s}(x)=\bigcup_{y \in F^{s}(x)} F^{t}(y) x \in I .
$$

Moreover, $x \notin F^{t}(x)$ for $t \neq 0$.

Proof. Fix an $x \in I$. Let $z \in F^{t} \circ F^{s}(x)$. Then there exists a $y \in F^{s}(x)$ such that $z \in F^{t}(y)$. This means that $\varphi(y)=\varphi(x)+s$ and $\varphi(z)=\varphi(y)+t$, which gives that $\varphi(z)=\varphi(x)+t+s$. Hence $z \in F^{t+s}(x)$. Similarly we prove the converse inclusion.

If $\varphi$ is a homeomorphism then Equation (1) defines the general form of continuous iteration groups such that $F^{1}(x) \neq x$ for $x \in I$.

If $\varphi$ is non-injective then s-v iteration group generated by $\varphi$ has very irregular properties and we will call this group singular. The purpose of this paper is the study of these "singularities."

Obviously the set-valued functions $F^{t}, t \in \mathbb{R}$ pairwise commute. This property is not transferible on the continuous selections of these set-valued mappings.

Let us assume that there exist $F^{u}, F^{v}$ with $\frac{u}{v} \notin \mathbb{Q}$ which possess homeomorphic commuting selections $f$ and $g$, that is $f(x) \in$ $F^{u}(x)$ and $g(x) \in F^{v}(x)$ for $x \in(a, b)$ and $f \circ g=g \circ f$. Then the generating function $\varphi$ satisfies the equations $\varphi(f(x))=\varphi(x)+u$ and $\varphi(g(x))=\varphi(x)+v$. Note that then $f, g$ are iteratively incommensurable, i.e.,

$$
f^{n}(x) \neq g^{m}(x), \quad n, m \in \mathbb{Z},|n|+|m|>0, x \in I,
$$

where $f^{n}$ denotes the $n$-th iterate of function $f$ and $f^{0}=i d$. Define

$$
L_{f, g}:=\left\{f^{n} \circ g^{m}(x), n, m \in \mathbb{Z}\right\}^{d} .
$$

The set $L_{f, g}$ does not depend on $x$ and either this set is the interval cl $I$ or $L_{f, g}$ is a nowhere dense perfect set in $I$ (see Zdun [7]). If the generating function $\varphi$ is continuous at least at one point of $L_{f, g}$ then it is continuous and it is monotonic (see [8]).

We have more

\section{THEOREM 2}

If $f$ and $g$ are commuting iteratively incommensurable homeomorphisms, then there exist infinitely many s-v iteration groups $\left\{F^{t}, t \in \mathbb{R}\right\}$ of type (1) such that $f(x) \in F^{1}(x)$ and $g(x) \in F^{s}(x)$ for an $s \notin \mathbb{Q}$, but the only one of them has a monotonic generating function $\varphi$. Then the generating function $\varphi$ is continuous and $\varphi\left[L_{f, g}\right]=\mathbb{R}$.

The proof is a simple consequence of Theorem 2 and Corollary 1 in Zdun [8].

The family $\left\{F^{t}, t \in \mathbb{R}\right\}$ is a single-valued iteration group if and only if $L_{f, g}=[a, b]$. Then $\varphi$ is strictly monotonic (see Zdun [8]).

In this paper we consider the case where $L_{f, g} \neq[a, b]$, that is $\left\{F^{t}: t \in \mathbb{R}\right\}$ is a proper set-valued iteration group.

In the next section we will consider the more general case.

\section{RESULTS}

Assume the following general hypothesis:

(H) $\varphi: I \rightarrow \mathbb{R}$ is a non-decreasing and non-injective surjection.

Then the function $\varphi$ is continuous and the values of $F^{t}$ are closed intervals or singletons. Denote by $\left\{I_{\alpha}, \alpha \in A\right\}$ a family of the intervals of constancy of $\varphi$. These intervals are closed. Put

$$
L^{*}:=I \backslash \bigcup_{\alpha \in A} I_{\alpha}
$$

and

$$
L:=I \backslash \bigcup_{\alpha \in A} \operatorname{Int} I_{\alpha} .
$$

Note that $\varphi_{\mid L^{*}}$ is strictly increasing, $\varphi\left[I_{\alpha}\right]$ are singletons and if $I_{\alpha}<I_{\beta}$ then $\varphi\left[I_{\alpha}\right]<\varphi\left[I_{\beta}\right]$.

It is easy to verify that the s-v iteration group $\left\{F^{t}: I \rightarrow\right.$ $c c[I], t \in \mathbb{R}\}$ generated by $\varphi$ has the following properties.

\section{PROPOSITION 1}

(i) For every $x \in I F^{t}(x)$ either is a closed proper interval $I_{\alpha}$ or a singleton belonging to $L^{*}$;

(ii) for every $x \in I$ the s- $v$ function $t \rightarrow F^{t}(x)$ is strictly decreasing, i.e., if $s<t$ then for every $u \in F^{s}(x)$ and $v \in F^{t}(x), u<v$;

(iii) for every $x \in I \bigcup_{t \in \mathbb{R}} F^{t}(x)=I$;

(iv) every s- $v$ function $F^{t}$ is constant on the intervals $I_{\alpha}$;

(v) if $s \neq t$ then $F^{t}(x) \cap F^{s}(x)=\emptyset$ for $x \in I$, that is the group $\left\{F^{t}, t \in \mathbb{R}\right\}$ is disjoint.

The conditions (i), (ii), (iii) characterize the interval-valued iteration groups. We have the following.

\section{PROPOSITION 2}

If an s-v iteration group $\left\{F^{t}, t \in \mathbb{R}\right\}$ satisfies conditions (i), (ii), and (iii), where $\left\{I_{\alpha}, \alpha \in A\right\}$ is a given family of closed disjoint proper intervals, then there exists a function $\varphi$ satisfying $(H)$ such that $F^{t}$ are given by the formula (1).

Proof. Define

$$
\mathcal{X}:=\left\{\{x\}, x \in L^{*}\right\} \cup\left\{I_{\alpha}, \alpha \in A\right\} .
$$

Let $x_{0} \in I$ and put $h(t):=F^{t}\left(x_{0}\right)$. Note that $h$ is a bijection from $\mathbb{R}$ onto $\mathcal{X}$. Define $\varphi$ by the following way: if $x \in I_{\alpha}$ for an $\alpha \in A$ then $\varphi(x):=h^{-1}\left(I_{\alpha}\right)$, if $x \in L^{*}$ then $\varphi(x):=h(\{x\})$. It is easy to see that $\varphi$ is a non-decreasing surjection of $I$ onto $\mathbb{R}$ constant on the intervals $I_{\alpha}$ and

$$
\varphi[h(t)]=t, \quad t \in \mathbb{R} .
$$

Since $F^{t} \circ F^{s}\left(x_{0}\right)=F^{t+s}\left(x_{0}\right)$ we have

$$
F^{t}[h(s)]=F^{t+s}\left(x_{0}\right)=h(s+t), \quad s, t \in \mathbb{R} .
$$


Hence

$$
\varphi\left[F^{t}(h(s))\right]=\varphi[h(s+t)]=s+t .
$$

Let $x \in I$. Then, by (iii), there exists an $s \in \mathbb{R}$ such that $x \in h(s)$. Hence $\varphi(x) \in \varphi[h(s)]=s$, thus $\varphi(x)=s$. This gives that $\varphi\left[F^{t}(x)\right] \subset \varphi\left[F^{t}(h(s)]=\varphi(x)+t\right.$, so

$$
\varphi\left[F^{t}(x)\right]=\varphi(x)+t .
$$

Since $F^{t}(x) \subset \varphi^{-1}\left[\varphi\left[F^{t}(x)\right]\right]$ we have $F^{t}(x) \subset \varphi^{-1}[\varphi(x)+t]$. Note that $\varphi^{-1}[\varphi(x)+t]$ is a singleton or equals to one of the intervals $I_{\alpha}$. If $F^{t}(x)$ is a singleton then, by (i), $F^{t}(x) \notin I_{\alpha}$ for any $\alpha \in A$. Thus $\varphi^{-1}[\varphi(x)+t]$ is not any of the intervals $I_{\alpha}$, so it is a singleton. If $F^{t}(x)$ is an interval $I_{\alpha}$, then $\varphi^{-1}[\varphi(x+t)]$ must be also the same interval. This gives equality $F^{t}(x)=\varphi^{-1}[\varphi(x+t)]$.

\section{PROPOSITION 3}

Let a family of set-valued function $F^{t}$ be given by (1), where $\varphi$ satisfies (H). Define

$$
f_{-}^{t}(x):=\inf F^{t}(x), f_{+}^{t}(x):=\sup F^{t}(x)
$$

for $t \in \mathbb{R}, x \in I$. Then

(i) the families $\left\{f_{-}^{t}, t \in \mathbb{R}\right\}$ and $\left\{f_{+}^{t}, t \in \mathbb{R}\right\}$ are iteration groups;

(ii) $f_{-}^{t}$ and $f_{+}^{t}$ for $t \in \mathbb{R}$ are non-decreasing discontinuous functions constant on the intervals of constancy of $\varphi$;

(iii) the mappings $t \rightarrow f_{ \pm}^{t}(x)$ are strictly decreasing;

(iv) $f_{-}^{t}[I] \subset L, f_{+}^{t}[I] \subset L, t \in \mathbb{R}$;

(v) $F^{t}(x)=\left[f_{-}^{t}(x), f_{+}^{t}(x)\right], t \in \mathbb{R}$.

Proof. (i) Fix an $x \in I$. Note that $f_{-}^{t}(x), f_{+}^{t}(x) \in F^{t}(x)$ since the sets $F^{t}(x)$ are closed. Hence, by Equation (1),

$$
\varphi\left(f_{ \pm}^{t}(x)\right)=\varphi(x)+t
$$

so $\varphi\left(f_{ \pm}^{t}\left(f_{ \pm}^{s}(x)\right)\right)=\varphi(x)+t+s=\varphi\left(f_{ \pm}^{t+s}(x)\right)$. This implies that

$$
f_{ \pm}^{t}\left(f_{ \pm}^{s}(x)\right) \in I_{\alpha} \text { and } f_{ \pm}^{t+s}(x) \in I_{\alpha}=F^{t+s}(x)
$$

for an $\alpha \in A$ or both belong to $L^{*}$, since $I_{\alpha}$ for $\alpha \in A$ are the intervals of constancy of $\varphi$. Obviously, in the second case, both values are equal. However, at the first case, $f_{+}^{t}\left(f_{+}^{s}(x)\right) \leq \sup I_{\alpha}=$ $f_{+}^{t+s}(x)$ and $f_{-}^{t+s}(x)=\inf I_{\alpha} \leq f_{-}^{t}\left(f_{-}^{s}(x)\right)$. On the other hand, putting $f_{+}^{s}(x)=: y$ we have that $f_{+}^{t}(y) \in I_{\alpha}$ and $f_{+}^{t}(y) \in F^{t}(y)$. Hence $F^{t}(y)=I_{\alpha}$ and $f_{+}^{t}(y)=\sup I_{\alpha} \geq f_{+}^{t+s}(x)$. This gives that

$$
f_{+}^{s}\left(f_{+}^{t}(x)\right)=f_{+}^{t+s}(x) .
$$

Similarly we prove that

$$
f_{-}^{s}\left(f_{-}^{t}(x)\right)=f_{-}^{t+s}(x)
$$

(iv) Proving (i) we have shown that $f_{ \pm}^{t}(x)$ either belong to $L^{*}$ or equals to one of the ends of the interval $I_{\alpha}$ which belong to $L$. Both cases give that $f_{ \pm}^{t}(x) \in L$.

The remaining assertions are the simple consequences of formula (Equation 1).
Let $\varphi$ be non-decreasing and non-injective surjection. Define the following family of functions

$$
\operatorname{Realm}(\varphi):=\left\{f: I \rightarrow I: \exists_{c_{f}} \forall_{x \in I} \varphi(f(x))=\varphi(x)+c_{f}\right\} .
$$

The index $c_{f}$ is uniquely determined. This allows us to define

$$
\text { ind } f:=c_{f} \text {. }
$$

As a particular case of Proposition 2.2 in Farzadfard and Zdun [9] we get the following

LEMMA 1

If $f \in \operatorname{Realm}(\varphi)$ then the following conditions are equivalent:

(i) $\varphi\left[L^{*}\right]=\varphi\left[L^{*}\right]+\operatorname{ind} f$;

(ii) $\varphi\left[I \backslash L^{*}\right]=\varphi\left[I \backslash L^{*}\right]+$ ind $f$;

(iii) $f\left[L^{*}\right]=L^{*}$;

(iv) $f$ maps each $I_{\alpha}$ into another one; moreover for every $I_{\beta}$ there exists $I_{\alpha}$ such that $f\left[I_{\alpha}\right] \subset I_{\beta}$.

Let $\varphi$ satisfy $(\mathrm{H})$ and define

$$
T:=\left\{t \in \mathbb{R}: \varphi\left[I \backslash L^{*}\right]+t=\varphi\left[I \backslash L^{*}\right]\right\} .
$$

If $T \neq\{0\}$, then $T$ is a countable Abelian subgroup of group $(\mathbb{R},+)$.

In fact, since $\varphi$ is constant in the intervals $I_{\alpha}$, we have $\varphi[I \backslash$ $\left.L^{*}\right]=\left\{\varphi\left[I_{\alpha}\right], \alpha \in A\right\}$. It is easy to see that this set is unbounded above and below thus it is infinite and, consequently, countable since the intervals $\left\{I_{\alpha}, \alpha \in A\right\}$ are pairwise disjoint.

\section{DEFINITION 1}

A subgroup $T$ given by Equation (5) is said to be a supporting group of the s-v iteration group $\left\{F^{t}: t \in \mathbb{R}\right\}$.

\section{THEOREM 3}

Let $T \neq\{0\}$ be a supporting group of $s-v$ iteration group $\left\{F^{t}: t \in \mathbb{R}\right\}$ generated by a function $\varphi$ satisfying $(H)$. Then

(i) if $t \in T$ then for every $x \in L^{*} F^{t}(x)$ is a single point and $F^{t}(x) \in L^{*}$;

(ii) if $t \in T$ then for every $\alpha \in A$ there exists $\beta \in A$ such that $F^{t}(x)=I_{\beta}$ for $x \in I_{\alpha}$;

(iii) if $t \in T$ then for every $\beta \in A$ there exists $\alpha \in A$ such that $F^{t}(x)=I_{\beta}$ for $x \in I_{\alpha}$;

(iv) if $F^{t}\left[L^{*}\right]=L^{*}$ then $t \in T$.

Proof. (i) By Equation (2) $f_{-}^{t}, f_{+}^{t} \in \operatorname{Realm}(\varphi)$, ind $f_{ \pm}^{t}=t$ for $t \in \mathbb{R}$ and $\varphi\left(f_{-}^{t}(x)\right)=\varphi\left(f_{+}^{t}(x)\right)$. By Lemma $1 f_{ \pm}^{t}(x) \in L^{*}$ for $x \in L^{*}$. Since $\varphi_{\mid I_{\alpha}}$ is injective $f_{-}^{t}(x)=f_{+}^{t}(x)$ for $x \in L^{*}$. Thus, by Proposition $3(\mathrm{v}), F^{t}(x)$ is a singleton belonging to $L^{*}$.

(ii) Let $x \in I_{\alpha}$. By Lemma $1 f_{ \pm}^{t}(x) \in I_{\beta}$ for a $\beta \in A$. Thus $F^{t}(x) \subset I_{\beta}$. If $F^{t}(x)$ is a singleton then, by Proposition 1 (i), $F^{t}(x)$ belongs to $L^{*}$, so $f_{ \pm}^{t}(x) \in L^{*}$, but this is a contradiction. Thus $F^{t}(x)$ is a proper interval, so $F^{t}(x)=I_{\beta}$.

(iii) Fix a $\beta \in A$. Since $\varphi\left[I_{\beta}\right]$ is a singleton and $\varphi$ is a surjection from $I$ onto $\mathbb{R}$ there exists an $x \in I$ such that $\varphi\left[I_{\beta}\right]=t+\varphi(x)$, that is $F^{t}(x)=I_{\beta}$. Suppose $x \in L^{*}$. Then, by Lemma $1, f_{ \pm}^{t}(x) \in L^{*}$, but 
this is a contradiction since $f_{ \pm}^{t}(x) \in F^{t}(x)=I_{\beta}$, so there exists an $\alpha \in A$ such that $x \in I_{\alpha}$.

(iv) Since $\varphi$ satisfies relation Equation (3) we have $\varphi\left[L^{*}\right]=$ $\varphi\left[F^{t}\left[L^{*}\right]\right]=\varphi\left[L^{*}\right]+t$, so, by Lemma $1, t \in T$.

Directly by Theorem 3 we get the following

\section{COROLLARY 1}

Let $T \neq\{0\}$ be the supporting group of the s-v group $\left\{F^{t}: t \in \mathbb{R}\right\}$ with generating function satisfying $(H)$. Then

(i) $T=\left\{t \in \mathbb{R}: \forall_{\omega \in A} \exists_{\bar{\omega} \in A} F^{t}\left[I_{\omega}\right]=I_{\bar{\omega}}\right\}$;

(ii) $T=\left\{t \in \mathbb{R}: \forall_{x \in L^{*}} F^{t}(x)\right.$ is a singleton $\}$;

(iii) $T=\left\{t \in \mathbb{R}: F^{t}\left[\mathrm{~L}^{*}\right]=\mathrm{L}^{*}\right\}$.

\section{DEFINITION 2}

A family of continuous mappings $\left\{f^{t}: I \rightarrow I, t \in T\right\}$ such that $f^{t} \circ f^{s}=f^{t+s}$ for $t, s \in T$ is said to be a T-iteration group.

Now we consider the problems connected with continuous selections of s-v iteration groups. The iteration groups $\left\{f_{-}^{t}, t \in\right.$ $\mathbb{R}\}$ and $\left\{f_{+}^{t}, t \in \mathbb{R}\right\}$ are the monotonic selections of s-v group $\left\{F^{t}, t \in \mathbb{R}\right\}$ that is $f_{ \pm}^{t}(x) \in F^{t}(x)$, but they are discontinuous.

Let $\varphi$ satisfies $(\mathrm{H})$ and $I_{\alpha}=:\left[a_{\alpha}, b_{\alpha}\right]$ for $\alpha \in A$ be the intervals of constancy of $\varphi$. For $t \in T$ define the affine mappings $q_{t, \alpha}:\left[a_{\alpha}, b_{\alpha}\right] \rightarrow I$ such that

$$
q_{t, \alpha}\left(a_{\alpha}\right)=f_{-}^{t}\left(a_{\omega}\right) \text { and } q_{t, \alpha}\left(b_{\alpha}\right)=f_{+}^{t}\left(b_{\alpha}\right) .
$$

For every $t \in T$ define the following mapping

$$
q^{t}(x):=\left\{\begin{aligned}
q_{t, \alpha}(x), & x \in I_{\alpha} \\
f_{+}^{t}(x), & x \in L^{*}
\end{aligned}\right.
$$

\section{LEMMA 2}

If $T \neq\{0\}$ is the supporting group of s-v group $\left\{F^{t}: t \in \mathbb{R}\right\}$ generated by a function satisfying condition $(H)$, then $\left\{q^{t}: I \rightarrow I, t \in T\right\}$ is a T-iteration group of continuous functions. Moreover, $q^{t}(x) \in F^{t}(x)$ for $t \in T$ and $x \in I$.

Proof. Note that $q_{t, \alpha}\left[I_{\alpha}\right]=F^{t}\left[I_{\alpha}\right]$ and $F^{t}\left[I_{\alpha_{1}}\right]<F^{t}\left[I_{\alpha_{2}}\right]$ if $I_{\alpha_{1}}<I_{\alpha_{2}}$. Hence, by Theorem 3, it follows that the mappings $q^{t}$ are strictly increasing surjections and, consequently, they are continuous.

It follows that for every $t, s \in T, q^{t} \circ q^{s}\left[I_{\alpha}\right]=q^{t}\left[F^{s}\left[I_{\alpha}\right]\right]=$ $F^{t}\left[F^{s}\left[I_{\alpha}\right]\right]=F^{t+s}\left[I_{\alpha}\right]=q^{t+s}\left[I_{\alpha}\right]$. Since the composition of affine functions is an affine function and there exists a unique increasing affine function mapping $I_{\alpha}$ onto the interval $F^{t+s}\left[I_{\alpha}\right]$ we get that $q^{t} \circ q^{s}=q^{t+s}$ on $I_{\alpha}$. Now it is easy to see that Proposition 3 implies our assertion.

\section{THEOREM 4}

If $s$-v group $\left\{F^{t}: t \in \mathbb{R}\right\}$ generated by a function satisfying condition $(H)$ has a non trivial supporting group $T$, then there exists infinitely many disjoint $T$-iteration groups $\left\{f^{t}, t \in T\right\}$ of continuous functions such that $f^{t}(x) \in F^{t}(x)$ for $t \in T$ and $x \in I$. $T$ is a maximal additive group with this property.
Proof. Let $\gamma: I \rightarrow I$ be a homeomorphism such that $\gamma(x)=x$ for $x \in L$ and for every $\alpha \in A \gamma\left[I_{\alpha}\right]=I_{\alpha}$. Put

$$
f^{t}:=\gamma^{-1} \circ q^{t} \circ \gamma, t \in T .
$$

It follows, by Lemma 2 , that $\left\{f^{t}, t \in T\right\}$ is a $T$-iteration group and $f^{t}(x) \in F^{t}(x)$.

Let $F^{t}$ have a continuous and strictly increasing selection $f$. Since for every $\alpha \in A, f\left[I_{\alpha}\right]$ is a proper interval, $F^{t}\left[I_{\alpha}\right]$ is also an interval. Thus, by Corollary $1, t \in T$.

Let us make the following assumptions.

(i) Let $L$ be a Cantor set in $I$, that is $L$ is a nowhere dense perfect set in $I=(a, b)$ and $a, b \in L$.

(ii) Let $I_{\omega}, \omega \in \mathbb{Q}$ be open pairwise disjoint intervals such that

$$
I \backslash L=: \bigcup_{\omega \in \mathbb{Q}} I_{\omega} .
$$

(iii) Let $\varphi: I \rightarrow \mathbb{R}$ be a Lebesgue function which lives on a set $L$ that is $\varphi$ is a continuous non-increasing surjection constant on $\mathrm{cl} I_{\omega}$ and, let cl $I_{\omega}$ be the maximal intervals of constancy of $\varphi$.

The conditions (i), (ii), and (iii) imply that $\varphi$ is continuous and

$$
\varphi[L]=\mathbb{R} .
$$

\section{THEOREM 5}

Let $T$ be the supporting group of s-v group $\left\{F^{t}: t \in \mathbb{R}\right\}$ generated by a function $\varphi$ satisfying condition $(H)$. If the group $T$ is acyclic then the set $L$ defined by (2) is a Cantor set and $\varphi$ is a Lebesgue function which lives on $L$.

Proof. By Lemma 2 the family of mappings $\left\{q^{t}, t \in T\right\}$ defined by Equation (6) is a disjoint $T$-iteration group. Denote by $L_{T}$ the set of limit points of the orbits $O(x)=\left\{q^{t}(x): t \in T\right\}$, i.e., $L_{T}=O(x)^{d}$. In Zdun [10] (see Th.1) it is proved that the set $L_{T}$ does not depend on $x$ and $L_{T}$ is either a Cantor set in $I$ or $L_{T}=[a, b]$ or $L_{T}=\{a, b\}$. Moreover, $L_{T}=$ $\{a, b\}$ if and only if $\left\{q^{t}, t \in T\right\}$ is a cyclic group (see [10] Theorem 2).

Since $q^{t}(x) \in F^{t}(x)$ we have $\varphi\left(q^{t}(x)\right)=\varphi(x)+t$ for $x \in I$. $L_{T} \neq[a, b]$. In fact, suppose that $L_{T}=[a, b]$. Fix an $x \in I$ and an interval $I_{\alpha}$. By the density of the orbit $O(x)$ there exist $u, v \in \mathbb{R}$ such that $u \neq v$ and $q^{u}(x), q^{v}(x) \in I_{\alpha}$. Hence $\varphi(x)+u=$ $\varphi\left(q^{u}(x)\right)=\varphi\left(q^{v}(x)\right)=\varphi(x)+v$ what is a contradiction.

By Proposition 1 (ii) and Lemma 2 the mapping $\Phi(t):=q^{t}$ is an isomorphism of $T$ onto the group $\left\{q^{t}, t \in T\right\}$. Thus $T$ is cyclic if and only if $\left\{q^{t}, t \in T\right\}$ is cyclic, so $T$ is cyclic if and only if $L_{T}=\{a, b\}$. Hence $T$ is acyclic if and only if $L_{T}$ is a Cantor set.

If $T$ is acyclic then $\varphi$ lives on $L_{T}$. Let $x \in L_{T}$ and $t \in T$. Then $q^{t}(x)=f_{+}^{t}(x) \in L$. Thus $O(x) \subset L$ and, consequently, $L_{T} \subset L$, so $L$ is also a Cantor set. By the assumption $\varphi$ lives on $L$, however by the definition of $q^{t} \varphi$ lives on $L_{F}$. Thus we get $L_{F}=L$. 


\section{THEOREM 6}

If $f, g$ are commuting, iteratively incommensurable homeomorphisms and $L_{f, g} \neq \mathrm{cl} I$, then $f$ and $g$ are embeddable in a non-extensible disjoint T-iteration group $\left\{f^{t}, t \in T\right\}$, where $T$ is a dense, countable subgroup of $\mathbb{R}$.

Proof. By Theorem 2 there exists an s-v iteration group $\left\{F^{t}\right.$ : $t \in \mathbb{R}\}$ with continuous non-decreasing generating function $\varphi$ such that $f(x) \in F^{1}(x)$ and $g(x) \in F^{s}(x)$ for an $s \notin \mathbb{Q}$ and $\varphi\left[L_{f, g}\right]=\mathbb{R}$. Since $L_{f, g} \neq \operatorname{cl} I, \varphi$ is a Lebesgue function which lives on $L_{f, g}$. Define $T$ by Equation (5). By Theorem $5 f$ and $g$ are embeddable in a $T$-iteration group $\left\{f^{t}, t \in T\right\}$. Since $1, s \in T$ the group $T$ is dense.

\section{DISCUSSION}

In this note we consider the relation between the iteration groups of monotonic functions and the interval-valued iteration groups. These groups are still poorly investigated.

In Section 2 we indicate a desirability of the generalization of classical iteration groups in the real case. It is known that not all commutable iteratively incommensurable homeomorphisms are embeddable in an iteration group. However, Theorem 2 shows that the embeddabilty is always possible for $s-v$ iteration groups.

Propositions 1 and 2 characterize s-v iteration groups of the form Equation (1). It is shown that, in our investigations, the

\section{REFERENCES}

1. Smajdor A. Iterations of multifunctions. Pr Nauk Uniw Slask Katowice (1985) 759:1-55.

2. Olko J. On indexed functions generated by families of functions. Aequat Math. (2014) 88:125-35. doi: 10.1007/s00010-013-0239-1

3. Olko J. Selections of an iteration semigroup of linear set-valued functions. Aequat Math. (1998) 56:157-68. doi: 10.1007/s000100050052

4. Łydzińska G. On lower semicontinuity of some set-valued iteration semigroups. Nonlinear Anal. (2009) 71:5644-54. doi: 10.1016/j.na.2009.04.051

5. Piszczek M. On multivalued iteration semigroup. Aequat Math. (2011) 81:97108. doi: 10.1007/s00010-010-0034-1

6. Zdun MC. On set-valued iteration groups generated by commuting functions. J Math Anal Appl. (2013) 398:638-48. doi: 10.1016/j.jmaa.2012.09.016

7. Zdun MC. Note on commutable functions. Aequat Math. (1988) 36:153-64. form Equation (1) of $\mathrm{s}-\mathrm{v}$ iteration groups are quite natural. Proposition 3 shows how $\mathrm{s}-\mathrm{v}$ iteration groups of the form Equation (1) determine iterations groups of non-decreasing functions which are not injective.

A key concept of the paper is the supporting group $T$ defined by Equation (5). If $T$ is non-trivial additive group then it is countable and the set of all intervals of constancy of the generating function $\varphi$ is also countable. Theorem 3 and Corollary 1 explain the meaning of the supporting group $T$. The restricted $\mathrm{s}-\mathrm{v}$ group $\left\{F^{t}: t \in T\right\}$ has a property that $\mathrm{s}-\mathrm{v}$ functions $F^{t}$ transform the intervals of constancy of the generating function $\varphi$ onto itself and the points from its complement, that is the set $L^{*}$, onto singletons in $L^{*}$. Moreover, Theorem 4 and Corollary 1 show that each s-v function $F^{t}$ for $t \in T$ has continuous selection $f^{t}$ such that family $\left\{f^{t}: t \in T\right\}$ forms a group. Moreover, any $F^{t}$ for $t \notin T$ has no continuous selection.

We have also proved that supporting group $T$ is acyclic if and only if the generating function $\varphi$ is a Lebesgue function which lives in a Cantor set.

The presented results may be helpful in the constructions of different iteration groups of non-decreasing functions.

\section{AUTHOR CONTRIBUTIONS}

MZ conceived the study and prepared the manuscript.

8. Zdun MC. On simultaneous Abel equations. Aequat Math. (1989) 38:163-77.

9. Farzadfard H, Zdun MC. On a limit formula for regular iterations. J Math Anal Appl. (2016) 443:947-58. doi: 10.1016/j.jmaa.2016.05.068

10. Zdun MC. On the orbits of disjoint groups of continuous functions. Rad Mat. (1992) 8:95-104.

Conflict of Interest Statement: The author declares that the research was conducted in the absence of any commercial or financial relationships that could be construed as a potential conflict of interest.

Copyright (C) 2016 Zdun. This is an open-access article distributed under the terms of the Creative Commons Attribution License (CC BY). The use, distribution or reproduction in other forums is permitted, provided the original author (s) or licensor are credited and that the original publication in this journal is cited, in accordance with accepted academic practice. No use, distribution or reproduction is permitted which does not comply with these terms. 